\title{
Efectos de la enseñanza interdisciplinaria en la educación ambiental sobre los conocimientos, valores y actitudes ambientales de estudiantes de segundo ciclo básico (Los Ángeles, Región del Biobío, Chile) ${ }^{1}$
}

\section{Effect of the interdisciplinary teaching of environmental education on knowledge, values and attitude of junior high school students (Los Ángeles city, Biobio Region, Chile)}

\author{
Laura Beatriz TORRES RIVERA, Nicol MESINA CALDERÓN, Brigite \\ SALAMANCA SALAZAR y Carla SEPÚLVEDA SEPÚLVEDA \\ Universidad de Concepción (Chile)
}

Recibido: Diciembre 2014

Evaluado: Marzo 2015

Aceptado: Mayo 2015

\section{Resumen}

Debido a la necesidad de implementar metodologías efectivas para la formación de ciudadanos con conciencia ambiental, se planteó esta investigación con el objetivo de evaluar el efecto de la enseñanza interdisciplinaria en la Educación Ambiental, sobre los conocimientos, valores y actitudes ambientales, de estudiantes de sexto curso básico de un establecimiento municipal de la ciudad de Los Ángeles, en Chile. Se relaciona la Educación Ambiental con las asignaturas Lenguaje-Comunicación y Matemática. Los instrumentos de medición, validados por un comité de expertos y sometidos a un análisis de fiabilidad. Se aplicaron antes y después de la intervención, permitiendo así medir el grado de cambio en conocimientos, valores y actitudes ambientales en los distintos tratamientos, los cuales fueron analizados estadísticamente por medio de tests no paramétricos. Los resultados indican que un grado mayor de interdisciplinariedad genera mejores conocimientos, valores y actitudes ambientales, por lo que se recomienda implementar estrategias interdisciplinarias en el aula al momento de realizar Educación Ambiental.

Palabras clave: educación ambiental, interdisciplinariedad, sexto curso básico, Chile.

\footnotetext{
${ }^{1}$ Investigación financiada por el proyecto UCO-1203.
} 


\begin{abstract}
Due to the need of implementing effective methodologies for the training of citizens with environmental awareness, this research was posed with the aim of evaluating the effect of the interdisciplinary teaching in Environmental Education on environmental knowledge, values and attitudes of sixth grade students teaching of a public school from Los Angeles city, in Chile. Environmental Education is related to the subjects of Language-Communication and Mathematics. The measurement instruments were validated by a committee of experts and subjected to a reliability analysis. These instruments were applied before and after the intervention, allowing measuring the degree of change in environmental knowledge, values and attitudes in the different treatments which were statistically analysed by means of nonparametric tests. Results indicate that a higher degree of interdisciplinarity generates better knowledge, values and attitudes towards the environment. Hence, it is recommended to implement interdisciplinary strategies in the classroom at the time of performing Environmental Education.
\end{abstract}

Keywords: environmental education, interdisciplinarity, $6^{\text {th }}$ grade, Chile.

En Chile la Educación Ambiental (EA) se ha definido en la ley N¹9.300 (2007) de Bases Generales del Medio Ambiente, como un proceso permanente de carácter interdisciplinario, destinado a la formación de una ciudadanía que reconozca valores, aclare conceptos, desarrolle habilidades y las actitudes necesarias para una convivencia armónica entre seres humanos, su cultura y su medio biofísico circundante. Lo anterior tiene relación con el hecho que la educación es un instrumento de transformación social, un camino viable para generar cambios favorables frente a los conflictos ambientales, no sólo creando consciencia, sino que facilitando el espacio de formación para personas intrínsecamente conscientes de los daños ambientales y de las posibilidades de solucionar problemas al respecto (Ministerio del Medio Ambiente, 2012a).

Sumado a lo anterior, la EA en Chile se encuentra considerada dentro de los Objetivos de Aprendizajes Transversales (OAT), los cuales tienen como finalidad que esta se desarrolle en cada una de las asignaturas comprendidas dentro del currículo, de manera transversal, especialmente en la de Ciencias Naturales, la cual comprende un pequeño espacio para tratar los contenidos implicados (Ministerio de educación, 2012b).

Si bien es cierto la EA está claramente establecida en el curriculum chileno, se considera que la metodología en que se imparte, no es lo suficientemente eficiente como para formar ciudadanos que contribuyan al desarrollo sustentable del país, cuya economía se basa esencialmente en la explotación de recursos naturales que la biosfera es capaz de ofrecer (SUMA, 2011). En la enseñanza de la EA, se ha detectado que los docentes presentan un desconocimiento de herramientas teóricometodológicas, carencia que viene desde la formación como educadores, debido a que no se imparte una enseñanza contextualizada en función de las temáticas ambientales, a partir de las cuales puedan transmitir una educación fundada en la sustentabilidad en las distintas asignaturas, sumado a una falta de conciencia ambiental de la población en 
general (SUMA, 2011) y de quienes tienen a su cargo la misión de cumplir los objetivos de la EA en el sistema educativo, los profesores (Condeza, 2012).

Probablemente, hay otros factores que están influyendo en que no se realice una eficiente EA en Chile. Estudios preliminares indican que el ambiente educativo de los establecimientos de enseñanza básica y media no estarían entregando las condiciones adecuadas para que se cumplan los objetivos de la EA; así como, las metodologías adecuadas, los profesores no estarían cumpliendo la transversalidad, interdisciplinariedad y la falta de tiempo y competencias para hacer EA estarían dentro de los factores más importantes para no realizarla (Coronado y Illanes, 2014; Benavides, Latoja y Novoa, 2014).

Frente a la crisis que esto genera, se demanda indudablemente un cambio, el que podría lograrse innovando en las metodologías de enseñanza, como la llevada a cabo por Álvarez y Vega (2010) al aplicar un método didáctico experimental en alumnos entre 12 y 16 años, o bien trabajar la EA desde un enfoque interdisciplinario, es decir, a partir de la integración efectiva de las disciplinas, la cual exhiba una intercomunicación y enriquecimiento recíproco de las diversas áreas, temáticas y conceptos para el logro de un nuevo conocimiento (Carvajal, 2010). Los investigadores coinciden en afirmar que la problemática ambiental es multi-causal, estructural, y por ende, es un problema que involucra a la totalidad de las ciencias y sociedades existentes, razón por la cual se tiene en consideración que la EA no es una disciplina independiente, ni una asignatura que debe ser tratada de manera aislada, sino que debe concebirse como una temática integral presente en todas las áreas del currículo (Fuentes et al, 2006).

Junto con lo anterior y desde el punto de vista de los educandos, ha de saberse que todas las personas aprenden de maneras diferentes y presentan distintas motivaciones y cualidades, razón por la cual, se cree que al tratar las temáticas ambientales utilizando las herramientas proporcionadas por otras disciplinas del aprendizaje, se generaría un mayor acercamiento de los alumnos hacia la ciencia y además, la adquisición de aprendizajes significativos, tanto del área de la EA, como de la asignatura con la cual se esté vinculando y enriqueciendo interdisciplinariamente (Carvajal, 2010).

Por esta razón, se considera que al enfrentar la EA de una manera interdisciplinaria, los estudiantes podrían identificarse con una o más posibilidades de aprendizaje, desarrollar sus potencialidades al mismo tiempo que adquieren una comprensión holística de la realidad, lograr mayor interés hacia el cuidado de la biosfera y por ende desarrollar aprendizajes significativos (Gómez, 2004).

Esto lleva a plantear la necesidad de utilizar en el aula una estrategia de enseñanza que combine distintos enfoques, de forma tal que permita a los alumnos hacer uso del pensamiento visual, espacial, al tratar integralmente las diversas áreas involucradas, las cuales conlleven a los estudiantes a reforzar y adquirir nuevos aprendizajes, como también, a desarrollar una conciencia ambiental que les permita modificar su actuar frente a los sistemas naturales (Delgado, 2009). 
Por lo anteriormente planteado, el objetivo de esta investigación fue evaluar la influencia de la enseñanza interdisciplinaria de la EA, sobre los conocimientos, valores y actitudes ambientales, de estudiantes de sexto básico de un establecimiento municipal de la ciudad de Los Ángeles, Región del Biobío, Chile.

\section{Metodología}

El diseño metodológico que se utilizó para realizar esta investigación fue del tipo cuasi-experimental. Lo anterior se abordó desde la perspectiva cuantitativa por medio del análisis de datos recogidos por encuestas y test de elaboración propia, cuyo objetivo principal fue pesquisar si hubo aprendizaje de EA a través de la interdisciplinariedad; así como, cambio en las actitudes y valores de los estudiantes en favor del medio ambiente (Hernández et al, 2006).

La elección del establecimiento fue de carácter no probabilístico. Se seleccionó un establecimiento municipal de la ciudad de Los Ángeles, Región del Biobío, Chile debido a su carácter mixto, su índice de vulnerabilidad medio y sus ponderaciones SIMCE (Sistema de Medición en la Calidad de la Educación); que lo colocan en un rango óptimo, lo que refleja que los alumnos no poseen un conocimiento acabado ni deficiente de los diversos contenidos.

Para el desarrollo de esta investigación, se usaron muestras no probabilísticas (Hernández et al, 2006). De un universo inicial de 88 estudiantes se establecieron 4 grupos, en los cuales se alcanzó un número de 22 alumnos por grupo, de los cuales el primero se trabajó como grupo control, razón por la cual no recibió ningún tratamiento, sino que se trataron los contenidos de acuerdo a la manera habitual en que suelen llevarse a cabo en los establecimientos; el segundo grupo se trabajó a partir de la integración de las áreas de Ciencias Naturales y Lenguaje y Comunicación; en el tercer grupo se involucraron las disciplinas de Ciencias Naturales y Matemática; finalmente, en el cuarto grupo se realizó una interdisciplinariedad mayor, ya que en él se integraron las asignaturas de Ciencias Naturales, Matemática, Lenguaje y Comunicación para la enseñanza de la EA.

El curso en el cual se llevó a cabo la investigación fue sexto año básico, con niños entre 11 y 12 años, en donde predominaban levemente los varones, los cuales, según los estadios de Piaget están finalizando las operaciones concretas, donde exhiben habilidad para resolver problemas de manera lógica concentrándose en el aquí y ahora; y comenzando las operaciones formales, donde demuestran un pensamiento abstracto, manejo de situaciones hipotéticas y pensamiento sobre las posibles soluciones a una determinada problemática (Papalia et al, 2012). Por otra parte, en este nivel los estudiantes presentan nociones básicas acerca del medio ambiente, no teniendo aún conocimientos tan profundos, por lo cual se estima que las actitudes se podrían modificar con mayor eficacia, que si se tratara con alumnos de niveles superiores.

Las temáticas tratadas y las razones por las cuales se seleccionaron se detallan a continuación: 
a) Calentamiento global: Problema que afecta a nivel mundial a los seres vivos y de gran realce debido a su origen humano (González, 2010).

b) Especies invasoras: Tema no muy abordado ni conocido por los estudiantes y la sociedad en general, pero que de igual manera trae grandes repercusiones a los ecosistemas (Quiroz et al, 2009).

c) La contaminación: Porque la ciudad de Los Ángeles, en los últimos años, ha sido muy afectada por esta temática, siendo destacada por los distintos medios de comunicación e investigaciones ambientales (Ulloa, 2011).

d) La eutrofización: Debido a que es una problemática ambiental no muy conocida por los estudiantes ni la población en general, pero que sin embargo se ha visto incrementada básicamente por la actividad humana (Parra, 1989).

Dichas problemáticas ambientales fueron conectadas interdisciplinariamente con las áreas de Lenguaje y Comunicación y Matemática. Para tratar las temáticas se diseñaron clases teórico-prácticas y guías interdisciplinarias de elaboración propia, de las cuales estas últimas se explican a continuación (para conocer un ejemplo de guías para los tratamientos interdisciplinarios ver la tabla 1 ):

1. Tratamiento grupo control: No se trabajaron guías interdisciplinarias.

2. Tratamiento grupo lenguaje: Se desarrollaron guías prácticas que relacionaban contenidos que son abordados en la asignatura de Lenguaje y Comunicación y Ciencias Naturales. En éstas, los alumnos debieron crear e identificar la estructura de textos literarios y no literarios, aplicando las reglas gramaticales a partir de una problemática ambiental dada.

3. Tratamiento grupo matemático: Se trabajaron guías prácticas que relacionaban contenidos tratados en la asignatura de Matemática y Ciencias Naturales, en las cuales los alumnos identificaron, analizaron y construyeron gráficos de distinta índole; calcularon y utilizaron números naturales, fracciones y porcentajes enfocados hacia las distintas problemáticas ambientales.

4. Tratamiento interdisciplinario mayor: Se utilizaron guías prácticas que relacionaban las tres asignaturas y contenidos previamente mencionados.

Dichos contenidos fueron seleccionados debido a la importancia que tienen en el nivel (ya que se abarcan en las distintas unidades en el transcurso del año), su presencia en la cotidianidad de los alumnos (ya sea en revistas, artículos, diarios, televisión, etc.) y su profundización en niveles posteriores.

Para evaluar conocimientos se elaboró un test de 24 preguntas de selección múltiple que abarcaban los problemas ambientales de importancia global y local, como energía, calentamiento global, introducción de especies, pérdida de la biodiversidad, eutroficación y contaminación atmosférica. Se le asignó el valor de 2 puntos a las respuestas correctas de dichas interrogantes y 0 puntos a aquellas respuestas que denotaban una carencia de conocimientos para la temática que estaba siendo examinada. La máxima puntuación de esta evaluación fue de 48 puntos. 
Las actitudes y valores ambientales se midieron a través de una encuesta de 26 aseveraciones y tres posibilidades de respuesta; siempre, a veces, nunca. Se le asignó 3 puntos al criterio "siempre", 2 puntos al criterio "a veces", 1 punto al criterio "nunca" y a aquellos criterios no contestados 0 puntos. La máxima puntuación de esta encuesta fue de 78 puntos.

\begin{tabular}{ll}
\hline GRUPO & DESCRIPCIÓN GUÍA \\
\hline Interdisciplinario & Como primera actividad los alumnos debieron leer un \\
Ciencia-lenguaje & documento con una noticia acerca de la invasión de \\
& especies. A partir de éste los alumnos respondieron cuatro \\
& preguntas, con la finalidad de trabajar su comprensión \\
& lectora. Posteriormente, se les entregaron a los alumnos \\
& cinco conceptos con los cuales debieron construir un \\
& cuento. Finalmente, construyeron un afiche acerca de los \\
& efectos de la invasión de especies. \\
\hline
\end{tabular}

Interdisciplinario Ciencia-matemáticas

La actividad se inició enfrentando al alumno a una serie de conceptos y definiciones, cada una antecedida por una fracción. Los alumnos identificaron las fracciones equivalentes y así unieron la definición y el concepto correspondiente. En la segunda actividad se le presentó una cuadrícula que representaba el $100 \%$ y en donde se les mostró con distintos colores la fracción para especies invasoras, endémicas y nativas. A partir de éste, debieron construir un gráfico de tortas y responder algunas preguntas. Como tercera actividad construyeron un gráfico de barras a partir de una tabla con cinco especies invasoras presentes en Chile y sus respectivas áreas de distribución.

Interdisciplinario

Ciencia-lenguajematemáticas
Para mantener el nivel de las actividades, se seleccionó la actividad de comprensión lectora y confección del afiche del tratamiento interdisciplinario Ciencia-Lenguaje y Comunicación y el trabajo de fracciones y construcción de gráficos a partir de los datos presentados en tabla, del tratamiento interdisciplinario Ciencia-Matemáticas.

Tabla 1. Descripción de una guía aplicada en los tratamientos interdisciplinarios usando la temática especies invasoras.

Ambos instrumentos fueron sometidos a una prueba de validación por 3 expertos pertenecientes al área de Ciencias de la Universidad de Concepción, Campus Los Ángeles; y fiabilidad, ya que dicho test y encuesta fueron aplicados a un grupo de alumnos de un establecimiento educacional con características similares al de la investigación interdisciplinaria. También, dichos instrumentos fueron sometidos al programa de fiabilidad Alfa de Cronbach. Los resultados alcanzados fueron 0,835 para 
el test de conocimientos ambientales y 0,815 para la encuesta de valores y actitudes. En función de lo anterior, se puede decir que ambos instrumentos mostraron una coherencia aceptable.

Para el análisis de los test de conocimientos, se consideraron los promedios $(60 \%$ de exigencia y nota mínima 2 debido a la exigencia del establecimiento según el reglamento de evaluación) alcanzados por los alumnos de manera general, por tratamiento y género; del mismo modo se trabajó en la encuesta de valores y actitudes ambientales, no obstante, los criterios enfocados a valores y actitudes se estudiaron de manera independiente.

Los resultados alcanzados en ambos instrumentos de evaluación fueron sometidos a un proceso de análisis, cuyo fin fue determinar si las muestras empleadas eran paramétricas o no paramétricas. Para ello se trabajó en el programa estadístico PAST (Barceló, 2007) y se sometieron los resultados a un test de normalidad y homocedasticidad, que indicaron que dichos resultados tenían un carácter no paramétrico. Por esta razón, se realizó un análisis basado en el test de Mann Whitney que compara solo dos variables, para de esta forma identificar si los conocimientos, valores y actitudes ambientales eran más significativos de acuerdo al grado de cambio percibido en los estudiantes (antes y después del tratamiento interdisciplinarios). Posteriormente se aplicó el test de Kruskal Wallis para ver qué tratamiento ( 2 o más) fue el que condujo a un cambio más importante en los alumnos (Hernández et al, 2006).

\section{Resultados}

Una vez finalizada la intervención interdisciplinaria en alumnos de sexto año básico se evidenciaron los siguientes resultados:

\section{Conocimientos ambientales}

Los post test de los distintos tratamientos mostraron cambios significativos en relación al pre test. La mínima alcanzada se percibió en el pre test $(3,3)$ y la máxima en el post test del tratamiento Interdisciplinario Mayor (5,3; gráfico 1).

Al comparar los resultados de conocimientos ambientales por género entre los test aplicados previo y posterior a la intervención, se obtuvo que los niños alcanzaron los promedios más altos, la mínima se apreció en el pre test de las niñas $(3,2)$ y la máxima en el post test del tratamiento interdisciplinario mayor $(5,3)$ tanto para niños como niñas, valor que resultó ser significativamente diferente al del pre test (gráfico 2).

\section{Valores y Actitudes Ambientales}

Los resultados indican que la metodología interdisciplinaria aplicada no varió en forma significativa los valores y actitudes en los tratamientos interdisciplinarios duales; sin embargo, si fue significativo el tratamiento interdisciplinario mayor, tanto en niñas como en niños (gráficos 3 y 4). 
El mayor promedio en valores ambientales se observó en la post encuesta del tratamiento interdisciplinario mayor, tanto en niños como en niñas (puntaje $=31,3$ y 27 respectivamente, gráfico 3). En relación a los puntajes de la pre y post encuesta de actitudes ambientales el promedio máximo se obtuvo en el tratamiento interdisciplinario mayor de ambos sexos (puntaje $=39,1$ en niños y 40,1 en niñas, gráfico 4$)$.

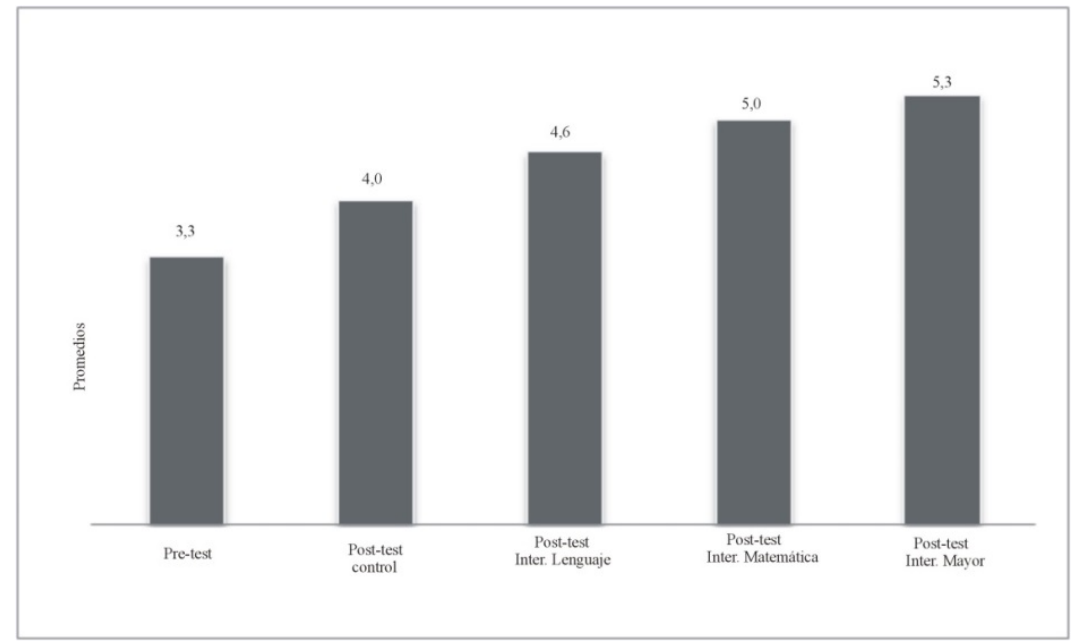

Gráfico 1. Promedios pre y post test de conocimientos ambientales para el grupo control y cada uno de los tratamientos.

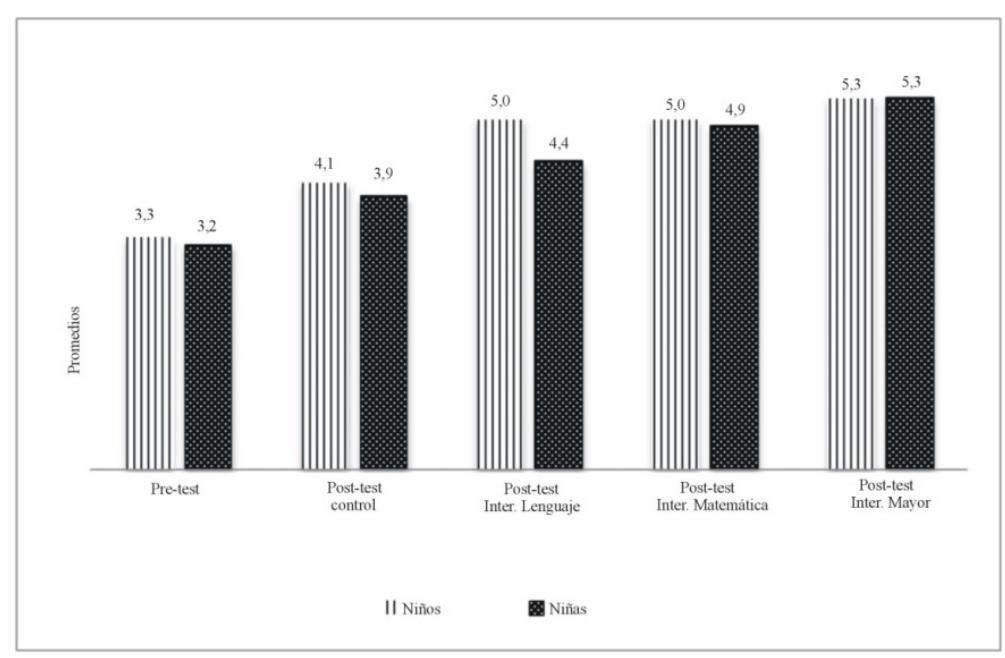

Gráfico 2. Promedios pre y post test de conocimientos ambientales por género para el grupo control y cada uno de los tratamientos. 


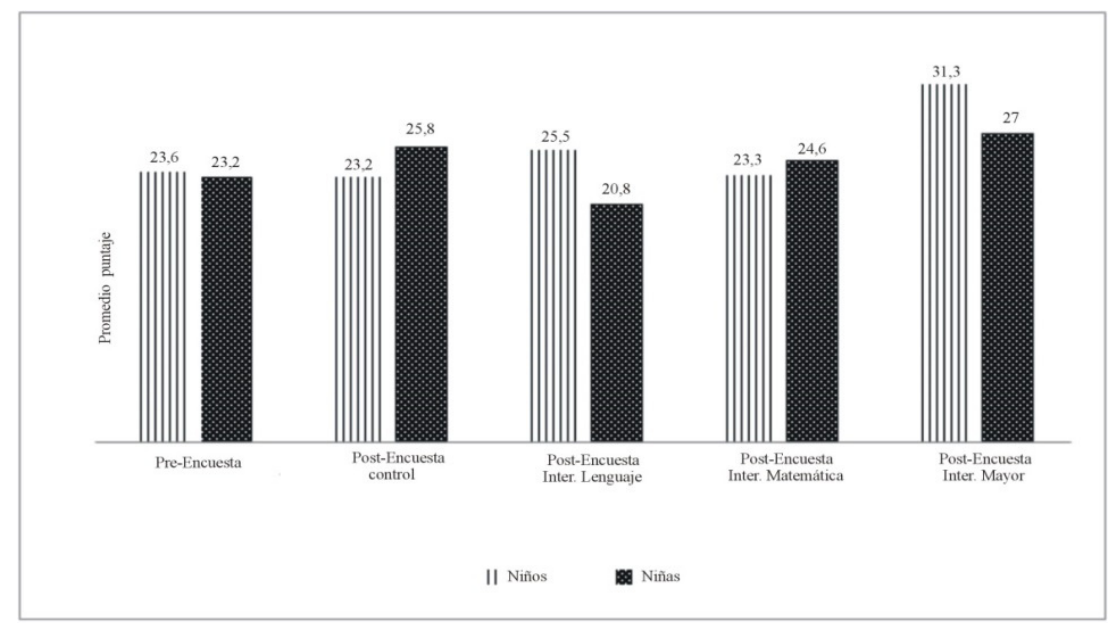

Gráfico 3. Promedio puntaje pre y post encuesta valores por género para el grupo control y cada uno de los tratamientos.

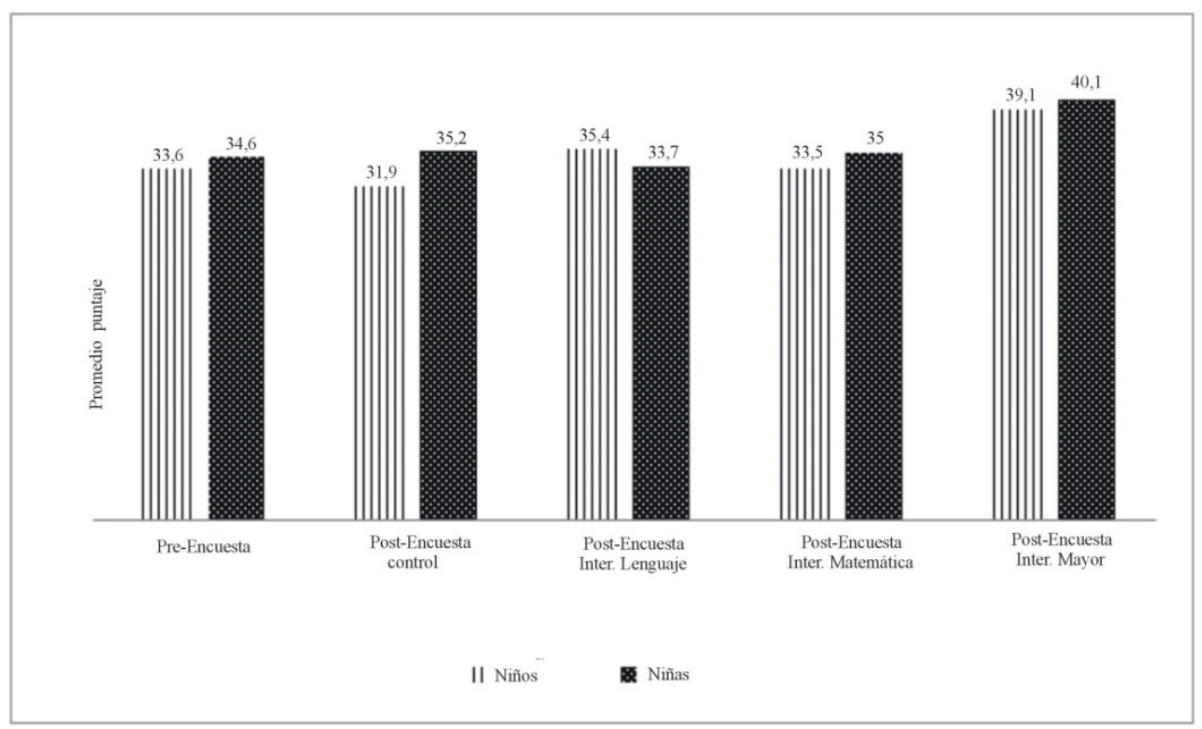

Gráfico 4. Promedio puntaje pre y post encuesta actitudes ambientales para el grupo control y cada uno de los tratamientos. 


\section{Discusión}

Variados esfuerzos se han realizado para generar cambios profundos y sostenidos a través del tiempo, respecto de las conductas de las personas, para el cuidado y protección del medio ambiente, de manera de lograr su preservación; es así como la educación, entendida como agente de cambio y promoción social adquiere un rol de gran importancia para generar y promover cambios en la sociedad (Martínez, 2010).

Dentro de esto, la EA, es concebida como el medio más efectivo para generar conciencia en la población sobre la necesidad de impulsar procesos enfocados en generar, evaluar y asegurar nuevas prácticas que garanticen la conservación y protección, con miras a una mejor calidad de vida tanto en el presente como para las generaciones futuras (Colón, 2011), lo cual cobra mayor importancia cuando las restricciones legales, la tecnología y desarrollo científico y las políticas que implementan los gobiernos no han sido suficientes (Aydim, 2012).

Sin embargo, las metodologías de enseñanza de la EA deben estar siendo constantemente revisadas y adaptadas a las distintas realidades educacionales. Los investigadores coinciden en afirmar que la problemática ambiental es multi-causal, estructural, y por ende, es un problema que involucra a la totalidad de las ciencias y sociedades existentes, razón por la cual se tiene en consideración que la EA no es una disciplina independiente, ni una asignatura que debe ser tratada de manera aislada, sino que debe concebirse como una temática integral presente en todas las áreas del currículo (Fuentes et al, 2006) y que por tanto la metodología con la que se entregan conocimientos, valores y actitudes también debe serlo.

En Chile, se decidió insertar la EA dentro de los Objetivos de Aprendizajes Transversales (OAT), los que por definición tienen un carácter comprensivo y general, cuyo fin es el fortalecer la formación ética de la persona, orientar el proceso de crecimiento, autoafirmación personal y la forma en que los educandos se relacionan con otros y el mundo (Ministerio del Medio Ambiente, 2012c). En este sentido, los OAT indican que la EA debe ser una temática mencionada, comentada y reflexionada en cada uno de los niveles y asignaturas impartidas en los establecimientos, en especial en los sectores de Ciencias Naturales, Historia y Ciencias Sociales (Ministerio del Medio Ambiente, 2009), lo cual implica el promover una EA, en donde los alumnos sientan que son parte de un problema que promueve su capacidad reflexiva y rige sus formas de actuar, haciéndolos de esta forma partícipes de su propio aprendizaje (Osses y Sánchez, 2004).

A pesar de los constantes esfuerzos del gobierno por tratar de promover una educación ambiental en donde los recursos sean valorados y utilizados en la cantidad justa, de manera de poder otorgarles a las futuras generaciones un patrimonio natural digno de apreciar, esta no ha sido lo suficientemente eficiente como para contribuir al desarrollo sostenible de Chile, cuya economía se basa principalmente en la explotación de los recursos naturales que la biota es capaz de ofrecerle, ya que se ha detectado que los docentes de la diversas especialidades, presentan un déficit en cuanto al conocimiento de herramientas teórico-metodológicas, que podría venir desde la formación inicial (Coronado y Illanes, 2014), que les impide integrar su disciplina con 
la EA (SUMA, 2011) y entregar una enseñanza significativa y globalizada. Por lo anterior, se hace necesario destacar que la efectividad de un método depende de la forma de presentación del tema, la seguridad del profesor, el conocimiento del mismo, la tendencia del alumnos y su disposición por ciertas materias o formas de enseñar (Jaramillo, 2006).

Sin embargo, no solamente son importantes las metodologías de enseñanza de la $\mathrm{EA}$, las cuales deben ser innovadoras e integradoras, sino que también cobra relevancia la seguridad del profesor en el dominio de los contenidos y la enseñanza de la EA. En este sentido, en un estudio realizado en 61 alumnos candidatos a profesor se vio que presentaban una alta confianza en la enseñanza de la EA, que no había diferencia en los resultados de género, pero que si influía el nivel académico, siendo los alumnos de quinto año los que alcanzaron mayor confianza en su desempeño, y el acercamiento de los alumnos a grupos o actividades ambientales (Çimen, Gökmen, Altunsoy, EKici y Yilmaz, 2011).

De lo anterior, se puede desprender la trascendencia de la formación inicial docente en el éxito de la enseñanza de la EA, la cual debe incorporar la EA e inculcar el apego de los estudiantes de pedagogía a la naturaleza, independiente de la especialidad, debido a que esto mejoraría su desempeño en el trabajo educativo de inculcar actitudes y valores ambientales.

Por otra parte, es necesario destacar que la transversalidad también aporta interdisciplinariedad, concepto que por definición intenta evitar el exceso de especialización en un área del aprendizaje, pues establece que una rama por sí sola no resulta una estrategia válida para abordar las múltiples dimensiones de la realidad. Por ello se considera que la temática ambiental (abordada desde una perspectiva transversal) no puede reducirse a un mero conocimiento sobre un campo, sino que debe abrirse a visiones amplias, donde el máximo de miradas se dirijan a obtener una visión integradora de la realidad (Chacón, 2006).

Tras la aplicación de los test, encuestas y metodología interdisciplinaria fue posible evidenciar una serie de cambios en los conocimientos, valores y actitudes de los alumnos de $6^{\circ}$ año que participaron en este estudio.

\section{Conocimientos ambientales}

Los estudiantes mostraron un cambio significativo en función de los conocimientos que presentaron antes y después del trabajo interdisciplinario (gráfico 1). Esta significancia se comprobó con los resultados obtenidos a partir del análisis de Mann Whitney que arrojó un valor de $\mathrm{p}=0,0001$. Entre los tratamientos interdisciplinarios, el más efectivo fue el Interdisciplinario Mayor, el cual integró Ciencias, Matemática y Lenguaje, lo que lleva a concluir que con la integración de tres disciplinas, los conocimientos se hacen más significativos para los educandos ya que existe un mayor impacto y comprensión de ellos desde las distintas perspectivas, pues como se sabe todas las personas son diferentes y presentan variadas formas de compresión que se pueden compensar y enriquecer mediante las prácticas integradoras 
de las distintas áreas del saber (Carvajal 2010). Lo anterior se comprueba con el análisis de kruskal Wallis, que evidenció que los tratamientos interdisciplinarios provocaron cambios muy significativos en los conocimientos de los estudiantes a diferencia del control (cuyo cambio fue mínimamente significativo), pues el p que presentaron fue inferior a 0,05 , siendo el más significativo y homogéneo el Interdisciplinario Mayor $(\mathrm{p}=3,464 \mathrm{E}-08)$.

Lo anterior denota que las prácticas interdisciplinarias son más efectivas que aquellas aplicadas tradicionalmente, las cuales son monodisciplinarias, ya que permiten otorgarle a los contenidos una visión holística de la realidad, dando un mayor sentido y significancia (Delgado, 2009).

Con respecto a los resultados por género, los varones presentaron un mejor promedio en el pre y post test en relación a las niñas, lo cual se podría atribuir al compromiso y motivación; así como, de sus habilidades en las asignaturas de Lenguaje-Comunicación y Matemática. En cuanto al efecto del tratamiento Interdisciplinario Mayor en niños y niñas, es posible mencionar que fue similar en ambos casos, pues los estudiantes promediaron un 5,3, sin embargo la dispersión de los resultados fue mayor en los varones (gráfico 2).

Con lo anterior se puede concluir que las prácticas interdisciplinarias son más efectivas que las aplicadas en la actualidad, ya que les permiten conocer e integrar los contenidos de mejor manera, independiente de la afinidad que los alumnos tengan con cierta asignatura (Carvajal, 2010), puesto a que las distintas áreas del aprendizaje se fusionan para mostrar una realidad única que puede ser captada significativamente desde diversas perspectivas, según las cualidades y desarrollo de los lóbulos cerebrales de los estudiantes, que por naturaleza hacen que los varones sean más lógicos y objetivos, mientras que las damas más emocionales y subjetivas (Salas, 2008). Por ello se estima conveniente realizar prácticas interdisciplinarias desde todas las áreas del saber, para que tanto educandos como profesores comprendan que todas las disciplinas se encuentran conectadas. Sumado a lo anterior, desarrollar en los alumnos que los problemas ambientales son fenómenos globales y por tanto deben ser enfrentados de una manera holística. De igual forma, al parecer y de acuerdo a los resultados de este estudio, mientras mayor es la integración de distintas disciplinas la obtención de resultados es más positiva.

\section{Valores y actitudes ambientales}

De forma general, la encuesta referida a los valores y actitudes ambientales de los estudiantes de sexto año básico, evidenció un cambio mínimo (gráficos 3 y 4); es decir, sin gran significancia $(\mathrm{p}>0,05)$ en dos de los tres tratamientos y que el tratamiento interdisciplinario mayor, entendido como la vinculación entre Ciencia, LenguajeComunicación y Matemática, permite incrementar los valores ambientales de los alumnos sometidos a dicha modalidad debido a que la diferencia con el pre-test fue significativa. Estos resultados coinciden con los encontrados por Thahong y Leopenwong (2014), quienes trabajando la EA con una metodología integral, la cual 
incorporó matemáticas, arte, tecnología, ciencia y lenguaje lograron promover la responsabilidad y cultivar valores ambientales intrínsecos.

Lo anterior, refuerza la idea de que es urgente implementar metodologías educativas innovadoras, destinadas a que los niños y niñas desarrollen en todos los niveles de su educación una permanente e incesante proyección de sus valores y actitudes ambientales. Sin embargo, para un efectivo desarrollo de la consciencia ambiental y posterior aplicación desde su comportamiento social, es necesaria la vinculación emocional-afectiva y, aún más, relacionar su contexto con la familiaridad pertinente requerida por la temática medio ambiental que les está afectando (Durán et al, 2007). Lo anterior, es comprobado por Álvarez y Vega (2010) quienes trabajando con estudiantes entre 12 y 16 años demostraron que el modelo didáctico experimental, de carácter investigativo, constructivista y basado en problemas ambientales del contexto cercano al estudiante incrementaba los conocimientos, actitudes e intención de conducta pro ambiente, probablemente explicado por la conexión emocional con su entorno.

Por otra parte, la actitud como constructo social, puede ser interpretada como la tendencia evaluativa hacia lo socialmente importante, es decir, los objetos relevantes tienden a que las actitudes coincidan con el comportamiento (Pacheco, 1994). Conceptualizando lo anterior con los resultados de la encuesta en el ámbito actitudes (gráfico 4) aplicada a los estudiantes, es posible destacar que las actitudes previas y posteriores de los alumnos varían levemente, no representando diferencia estadísticamente significativa $(\mathrm{p}>0,05)$ en los tratamientos interdisciplinarios duales.

Esta leve diferencia se puede sustentar en base a que las actitudes proambientales en los individuos constituyen un apego y apropiación de una identidad natural, que es posible observar por medio de procesos progresivos en el tiempo y son esenciales para el bienestar y comportamiento favorable individual hacia el desarrollo sustentable (Moser, 2003). La aplicación de talleres esporádicos no permite evidenciar resultados importantes en el área valórico-actitudinal, más bien, se requiere un trabajo de más tiempo y a través de todos los niveles de educación de los estudiantes. En este sentido, es difícil decir cuál es el tiempo mínimo requerido para lograr cambios en las actitudes, pero Álvarez y Vega (2010) lograron en un año de trabajo un incremento significativo en la intención de conducta.

Otro aspecto que podría estar infiriendo en los resultados, es la importancia de la emoción y la motivación en la toma de decisión, ya que los agentes implicados requieren de un proceso más complejo para desarrollar las distintas áreas cognitivas y socio-emocionales que le permitirán ejercer un rol a favor del medio ambiente (Parales y Vizcaíno, 2007). Concluyendo así, que una intervención interdisciplinaria, mediante talleres esporádicos de temáticas ambientales, mejoraría mínimamente las actitudes de los estudiantes.

A pesar de lo anterior, el tratamiento Interdisciplinario Mayor provocó un cambio significativo, debido a que niños y niñas aumentan su puntuación media en 5,5 con respecto a la pre-encuesta. Por consiguiente, la interdisciplinariedad mayor permitió modificar las actitudes de estudiantes sometidos a dicha modalidad; probablemente, 
promoviendo cambios importantes al lograr una efectiva conexión de las distintas áreas del currículum, independientemente del género (Castañer y Trigo, 1998).

Finalmente, el estudio realizado tuvo la limitación de haber sido implementado en sólo un nivel de enseñanza y por tanto surge la pregunta, ¿se obtendrían iguales resultados en otros niveles del sistema educativo chileno? Otro factor en contra podría haber sido el corto periodo de aplicación; sin embargo, se lograron resultados positivos en cuanto a la adquisición de conocimientos, actitudes y valores en el tratamiento interdisciplinario mayor; por lo cual, se hace necesario implementar metodologías innovadoras de enseñanza de la EA que permita fortalecer o desarrollar valores y promover actitudes en beneficio de una sociedad sustentable (Grisolía, 2008).

\section{Conclusiones}

A partir de los resultados anteriormente expuestos (valores y actitudes ambientales), es posible concluir que:

- El tratamiento Interdisciplinario Mayor fue el más efectivo y significativo al momento de generar mayor compresión de las distintas problemáticas ambientales, debido a que los educandos tuvieron más posibilidades de incrementar sus conocimientos científicos a partir de otras áreas del aprendizaje, a diferencia de aquellas prácticas en donde se daba escasamente.

- En el tratamiento interdisciplinario mayor, tanto niños como niñas reflejan un incremento de valores y actitudes ambientales. Ahora bien si establecemos una diferencia entre niños y niñas, estas últimas poseen y demuestran contribuir significativamente al cuidado de la biósfera.

Si se compara la efectividad interdisciplinaria entre el tratamiento Matemático y de Lenguaje - Comunicación, es posible mencionar que los contenidos más eficientes fueron los primeros, ya que provocaron un mayor impacto en los alumnos, quienes en todo momento tuvieron una media superior al otro tratamiento.

\section{Agradecimientos}

Esta investigación fue financiada por el proyecto UCO-1203. Las autoras expresan su agradecimiento al Dr. Francisco Brovelli y al Mg Jonathan Guzmán del Departamento de Ciencias Básicas, Escuela de Educación, Campus Los Ángeles de la Universidad Concepción por los valiosos comentarios que permitieron mejorar el manuscrito.

\section{Referencias bibliográficas}

ÁLVAREZ, P. y VEGA, P. (2010). Developing sustainable environmental behavior in secondary education students (12-16) Analysis of a didactic strategy. Procedia Social and Behavioral Sciences, 2, 3568-3574. 
AYDIM, S. (2012). On the role of intrinsic value in terms of environmental education. Procedia Social and Behavioral Sciences, 47, 1087-1091.

BARCELÓ, J. (2007). Arqueología y Estadística. Introducción al estudio de la variabilidad de evidencias arqueológicas. Universitat Autójnoma de Barcelona Servel de Publicacions.

BENAVIDES PEÑA, J. LATOJA VILLOUTA, C. Y NOVOA CONTRERAS, E. (2014). Evaluación de los tres ejes principales de la educación ambiental en profesores de ciencias naturales de primer y segundo ciclo de educación básica de establecimientos municipales de la ciudad de Los Ángeles. (Tesis inédita de pregrado). Universidad de Concepción, Campus Los Ángeles, Los Ángeles.

CARVAJAL, Y. (2010). Interdisciplinariedad desafío para la educación superior y la investigación. Luna Azul ISSN, 31, 156-169.

CASTAÑER, M. y TRIGO, E. (1998). La interdisciplinariedad en la educación secundaria obligatoria. Propuestas teórico-prácticas. (2 ${ }^{\mathrm{a}}$. Ed.). España: INDE publicaciones.

CHACÓN, J. (2006). Educación, interdisciplinariedad y pedagogía. Pampedia, 3, 22.

ÇIMEN, O. GÖKMEN, A. ALTUNSOY, S. EKICI, G. y YILMAZ, M. (2011). Analysis of biology candidate teacher'self-efficacy beliefs on environmental education. Procedia Social and Behavioral Sciences, 15, 2549-2553.

COLÓN, A. (2011). La Educación Ambiental: una herramienta para la protección y conservación del entorno. Revista $360^{\circ}, \mathrm{N}^{\circ} 6,1-5$.

CONDEZA ABARZÚA, K. (2013). Estudio de la relación entre el grado de conciencia ambiental en docentes y estudiantes de pedagogía y su área de especialidad: ipuede un docente hacer educación ambiental sin conciencia ambiental? (Tesis inédita de pre-grado). Universidad de Concepción, Campus Los Ángeles, Los Ángeles.

CORONADO GONZÁLEZ, E. Y ILLANES RUIZ, N. (2014). Estudio de la presencia de la Educación Ambiental transversal e interdisciplinaria en el ejercicio docente en la enseñanza media en establecimientos educacionales de la ciudad de Los Ángeles. (Tesis inédita de pre-grado). Universidad de Concepción, Campus Los Ángeles, Los Ángeles.

DELGADO, R. (2009). La integración de los saberes bajo el enfoque dialéctico globalizador: La interdisciplinariedad y transdisciplinariedad en educación. Investigación y Postgrado, 3, 14-27.

DURÁN, M. AlZATE, M. LÓPEZ, W. y SABUCEDO, J. (2007). Emociones y comportamiento pro-ambiental. Revista Latinoamericana de Psicología, 39, 287296.

FUENTES, L. CALDERA, Y. MENDOZA, I. (2006). La transversalidad curricular y la enseñanza de la educación ambiental. Orbis/Ciencias Humanas, 4, 41. 
GÓMEZ, J. (2004). Neurociencia cognitiva y educación. Perú.

GONZÁLEZ, E. ARIAS, M. (2010). Balance y perspectivas de la Educación Ambiental en Chile e Iberoamérica. El programa Internacional de Educación Ambiental: Institucionalización y Hegemonía, 59-69.

GRISOLÍA, M. (2008). La interdisciplinariedad en la enseñanza de las ciencias. Ciencia y Educación. 1-5.

HERNÁNDEZ, R. FERNÁNDEZ-COLLADO, C. y BAPTISTA, P. (2006). Metodología de la investigación. (4 ${ }^{\mathrm{a}} \mathrm{Ed}$.). México: McGraw-Hill.

JARAMILLO, J. (2006). ¿Cómo enseñar la educación ambiental? Recuperado de http://lunazul.ucaldas.edu.co/index.php?option $=$ com_content\&task $=$ view\&id $=273 \&$ Itemid $=273$.

MARTÍNEZ, J. (2010). Balance y perspectivas de la Educación Ambiental en Chile e Iberoamérica. Educación. Educación superior, globalización y Educación Ambiental. Chile.

MINISTERIO DEL MEDIO AMBIENTE. (2012a) ¿Qué es Educación Ambiental?

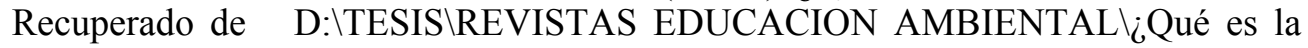
Educación Ambiental Chile Desarrollo Sustentable.mht

MINISTERIO DEL MEDIO AMBIENTE. (2012b). Eje transversal: Educación Ambiental. Recuperado de http://www.mma.gob.cl/1304/w3-propertyvalue16234.html

MINISTERIO DEL MEDIO AMBIENTE. (2012c). Eje transversal: Educación Ambiental. Recuperado de http://www.mma.gob.cl/1304/w3-propertyvalue16234.html

MINISTERIO DEL MEDIO AMBIENTE. (2009). Política Nacional. De Educación para el Desarrollo Sustentable. Revista de Educación Ambiental. 10, 23-25.

MOSER, G. (2003). La psicología ambiental en el Siglo 21: El desafío del desarrollo sustentable. Revista de Psicología de la universidad de Chile, XII, 11-17.

OSSES, S. SANCHEZ, I. (2004). Transversalidad curricular de la educación ambiental. Revista de educación ambiental, 3, 42-43

PACHECO, F. (1994). Actitudes. Revista Eúphoros. Recuperado de http://dialnet.unirioja.es/servlet/revista?codigo $=6432$

PAPALIA, D. DUSKIN, R. y MARTORELL, G. (2012). Desarrollo humano. (12 Ed.). México: Mc Graw-Hill.

PARALES, C. y VIZCAÍNO, M. (2007). Las relaciones entre actitudes y representaciones sociales: elementos para una integración conceptual. Revista Latinoamericana de Psicología, 39, 351-361.

PARRA, O. (1989). La Eutroficación de la Laguna San Pedro, Concepción, Chile: un caso de estudio. Ambiente y Desarrollo, 1, 117 - 136. 
QUIROZ, C. PAUCHARD, A. MARTICORENA, A. CAVIERES, L. (2009). Manual de plantas invasoras del centro-sur de Chile. Chile: LIB.

SALAS, R. (2008). Estilos de aprendizaje a la luz de la neurociencia. Primera edición. Cooperativa Editorial magisterio, Colombia.

SUMA (Sustentabilidad y Medio Ambiente). (2011). Actuales falencias en el aporte de la universidad al tema ambiental y desarrollo sustentable. Recuperado de http://sumaserver.gotdns.com/suma/?page_id=518.

TRATHONG, K. Y LEOPENWONG, S. (2014). The development of environmental education activities for forest resources conservation. Procedial Social and Behavioral Sciences, 116, 2266-2269.

ULLOA, G. (2011). Ecologistas califican de positiva pero tardía la declaración de zona saturada en Los Ángeles. Biobío, Chile. Obtenido el 20 de Febrero de 2013 desde http://www.biobiochile.cl/2011/05/26/ecologistas-califican-de-positiva-pero-tardiala-declaracion-de-zona-saturada-en-los-angeles.shtml

\section{Correspondencia con los autores}

Laura Beatriz TORRES RIVERA

Universidad de Concepción, Campus Los Ángeles

Juan Antonio Coloma 0201,

Los Ángeles, Región del Biobio, Chile

e-mail: latorres@udec.cl

Nicol MESINA CALDERÓN

Universidad de Concepción, Campus Los Ángeles

Juan Antonio Coloma 0201

Los Ángeles, Región del Biobio, Chile

e-mail: nmesina@udec.cl

Brigite SALAMANCA SALAZAR

Universidad de Concepción, Campus Los Ángeles

Juan Antonio Coloma 0201

Los Ángeles, Región del Biobio, Chile

e-mail: bsalamanca@udec.cl

Carla SEPÚLVEDA SEPÚLVEDA

Universidad de Concepción, Campus Los Ángeles

Juan Antonio Coloma 0201

Los Ángeles, Región del Biobio, Chile

e-mail: carlsepulveda@udec.cl 